\title{
Three Dimensional Finite Element Analysis of Radial-flow Impeller Temperature Field
}

\author{
Liangwei Zhong \\ CAD center, University of Shanghai for Science and Technology \\ Shanghai 200093, China \\ Tel: 86-21-55276487Ｅ-mail:zlvcad@126.com \\ Kangmin Chen \\ College of Power Engineering \\ University of Shanghai for Science and Technology \\ Shanghai 200093, China \\ Tel: 86-21-55274627Ｅ-mail: chenkm@usst.edu.cn \\ Jing Ni \\ Business School \\ University of Shanghai for Science and Technology \\ Shanghai 200093, China \\ Tel: 86-21-55276487Ｅ-mail:nijing501@126.com \\ Anyang $\mathrm{He}$ \\ CAD center, University of Shanghai for Science and Technology \\ Shanghai 200093, China \\ Tel: 86-21-55276487Ｅ-mail:heay@yahoo.com
}

Supported by Shanghai Leading Academic Discipline Project (T0502) and Shanghai Education Committee (05EZ30)

\begin{abstract}
The technique of three dimensional solid element model and assembly was used to determine the temperature field of radial-flow impeller. The FEM software Cosmos was applied to analyze model system, and the precise analytic results were obtained. The results show that the analytic model can reflect the stead-state and transient temperature field characteristics of impeller directly, and thus can be worthy reference to analyze and calculate the temperature field of impeller in engineering design.
\end{abstract}

Keywords: Runoff blade wheel rotor, Three dimension, Assembly, Finite element, Temperature field

Turbine wheel and compressor wheel are the most important elements of a gas turbine. The analysis of a gas turbine's temperature field characteristics under the circumstances of coldness, heat, start ,stop and steady working condition is vital to the study of fatigue and service life loss of a rotor. Because the geometry shape of an impeller is complicated, the two-stage analysis method and the direct computing method of an impeller are often used in engineering practice. The two-stage analysis method is relatively effective for computing an impeller with complicated geometry shape, but the computing process is complex and the work capacity is large, thus the deviation of the computing result is easily made. The advantage of the direct computing method is the effectiveness and accuracy of it is higher. However, both of these two computing methods take no consideration of the local variation of a body wheel's structure and they can not exactly reflect the distribution of the temperature field of every part, either. The structural finite element computing method takes the complexity and every kind of boundary and constraint condition of the blade wheel rotor's structure into full account, so the computing of the complicated structure's temperature field is effectively achieved and the temperature characteristics of the blade wheel rotor's every part is clearly reflected. Moreover, the assembly finite element computing method makes enough allowance for the practical situation of loading and thermal transmission and 
the analytic result of the whole rotor system is obtained. In this paper, Solidworks was used in modeling and the FEM software Cosmos was applied to wholly analyze the turbo rotor of the micro gas turbine system. Because of the complicated structure and the difficulty of dividing the net element by using the rotation symmetry method, in this paper, the finite element analysis towards the whole blade wheel is made.

\section{The analytic model and the computing method of the steady and transient temperature field of an blade wheel rotor}

The rotor heat analytic system is used for computing the temperature distribution characteristics of a system and the other thermo physical parameters, such as heat, heat gradient, heat-flow density (heat flux) and so on.

As long as a sealed system is concerned, according to the first law of thermodynamics, that is the law of conservation of energy, we can have the following equation:

$Q-W=\Delta U+\Delta K E+\triangle P E$

In this equation,

$\mathrm{Q} \longrightarrow$ heat

$\mathrm{W}$ - work

$\Delta U \_$_ internal energy of a system

$\triangle K E$ _ _ kinetic energy of a system

$\triangle P E \_$potential energy of a system

As long as most of the engineering thermal transmission problems are concerned:

$\triangle K E=\triangle P E=0$

Usually, the work is taken as: $W=0$, so we can get:

$Q=\Delta U$

As for the steady situation, $Q=\Delta U=0$, and this means that the heat flowing into the system and the heat flowing out of the system are the same. As for the transient thermal analysis, $q=\frac{d U}{d t}$, and this means that the heat transmission rate $q$ is equal to the change of the internal energy of a system.

\subsection{Steady thermal analysis}

In engineering field, equipment is said to be in the condition of steady thermal transmission when it is operating steadily. Besides this, when the problem of thermal transmission grading from steady to transient is dealt with, the steady thermal analysis should be taken as the last step of the transient thermal analysis and the condition of the system in the steady can be determined.

When the equipment is in the steady, the total of the heat quantity generated by the system itself and the heat quantity flowing into the system is equal to the heat quantity flowing out of the system. Thus, we can get $q_{\text {in }}+q_{\text {gen }}-q_{\text {out }}=0$. In the process of the steady, the temperature at any pitch point is invariable with time.

The equation of energy balance of the steady thermal analysis can be expressed in the form of matrix like this:

$[K]\{T\}=\{Q\}$

In this equation, $[\mathrm{K}]$ refers to a conduct matrix including coefficient of heat conductivity, coefficient of convective heat transfer, radiation factor, shape factor and so on. $\{\mathrm{T}\}$ refers to pitch point temperature vector. And $\{\mathrm{Q}\}$ refers to pitch point heat flow rate vector including thermogene.

$[\mathrm{K}],\{\mathrm{T}\}$ and $\{\mathrm{Q}\}$ can be obtained through using model geometric parameter, material thermal characteristic parameter and boundary conditions.

$[K]_{e}\{T\}_{e}=\{Q\}_{e}$, the equation of every unit, superposed according to its sequence number can form the entity matrix as $[K]_{n \times n}\{T\}=\{P\}_{n}$

\subsection{Transient thermal analysis}

During the process of unsteady, the temperature field is changing with time. As long as the problem of un-periodic thermal transmission is concerned, the temperature inside the objects going up or down constantly with time will go to the temperature of the environment and achieve the balance ultimately after a long time.

The basic steps of the transient thermal analysis are similar to those of the steady thermal analysis, and the main 
difference between them is that the load of the transient thermal analysis is changing with time. In order to express the load changing with time, the load-time curve should be divided into load steps firstly. Every inflection point of the load-time curve is regarded as a load step, the load value and the time value of every load step should be defined to every load step, and at the same time, the load step should be clearly differentiate from gradual changing to step-changing.

In the process of the transient heat transfer, the temperature, heat flow rate, heat flow boundary conditions and the inner energy of the system is changing with time. According to the principle of conservation of energy, we can get

$[C]\left\{T^{\&}\right\}+[K]\{T\}=\{Q\}$

In this equation, $[\mathrm{K}]$ refers to a conduct matrix including coefficient of heat conductivity, coefficient of convective heat transfer, radiation factor, shape factor and so on. [C]refers to specific heat capacity matrix. $\{\mathrm{T}\}$ refers to pitch point temperature vector. $\left\{T^{\&}\right\}$ refers to temperature vs. time derivate. And $\{\mathrm{Q}\}$ refers to pitch point heat flow rate vector including thermogene.

During the concrete heat-transfer process, the material thermal characteristic parameter and boundary conditions might be changing with time, then the above equation can be changed as:

$[C(T)]\left\{T^{\&}\right\}+[K(T)]\{T\}=[Q(T)](5)$

This kind of problems can also be taken as non-linear thermal analysis which mainly includes the following instances:

a. material characteristics is the function of temperature, such as $\mathrm{K}(\mathrm{T}), \mathrm{C}(\mathrm{T})$ and so on.

b. boundary conditions are changing with time, eg. $\mathrm{h}(\mathrm{T})$.

c. radiative heat transfer is involved.

\section{Finite element load simplification and result analysis}

\subsection{Temperature field analysis of flat blower blade wheel composite structure}

As Diagram 1 shows, rotor mainly includes turbo-expander wheel, compressor blade, axis of rotation and other parts.

Mainly because of the contact between turbo-expander wheel and compressor blade, the heat conduction exists among the parts inside the rotor. As the contact surfaces incline to each other or unhitch slightly for the air pressure, there are air gap units between the contact surfaces, by which the turbo-expander wheel and compressor blade form a holistic heat transfer system.

According to the literature, the rotor heating power boundaries can be divided into three types: the first kind of boundary condition, the third kind of boundary condition and adiabatic boundary condition.

a. the first kind of boundary condition

The first kind of boundary condition refers that the boundary temperature value of the appointed object is already known. The pitch point temperature of the axis surface is obtained according to the gas temperature of the air bearing.

b. the third kind of boundary condition

The third kind of boundary condition refers to the boundary condition under which the gas temperature of the rotor surfaces and the hear transfer coefficient are already known. The heat convection between high-speed gas flow or air flow and the water passage surface is the central mode of heat transfer of the rotor.

c. adiabatic boundary condition

Other than the above tow types of boundary conditions, all the other boundary conditions are presupposed as transferring no heat with the environment.

\subsection{Finite element result and analysis}

The temperature field in steady is illustrated by Diagram 2. The heat quantity is transferred through turbo-expander wheel to compressor blade. The temperature of the part of the turbo-expander wheel close to the interval channel is the highest, and the temperature of compressor blade close to the interval channel is similar to the compressed air. The temperature is changing as the shape of a stairway from the turbo-expander wheel to compressor blade.

Transient temperature field in different hour of the day is illustrated as Diagram 3 and Diagram 4. The total time is calculated as 140 seconds, and the delta time is 2 seconds. Diagram 3 and 4 show the temperature field at time of 80 s and 140s separately. The temperature distribution tendency is the same as that of the temperature field in steady. The highest temperature at the time of $80 \mathrm{~s}$ is only $222.5^{\circ} \mathrm{C}$, while the highest temperature after $140 \mathrm{~s}$ is $544.3^{\circ} \mathrm{C}$ which is close to the temperature of fuel gas, then the temperature grades into the values of the temperature field in steady. 


\section{Conclusion}

1) Because the geometry and operating condition of blade wheel rotor are complex, through the analysis by using three dimensional finite element model, the temperature distribution of it can be obtained effectively and exactly. The analyzing result is visualizable and engineering precise.

2) The blade graticule is divided more densely in calculation, and a blade consists at least three units. The precision of calculation can be improved.

3) The analytic technique of the finite element in assembly makes the model closer to the practical working condition and simplifies the loading difficulty of single element model.

\section{References}

ANSYS guide of thermal analysis.

Шубенко-шубин,Л.А. Intension of Steam Turbine. China Machine Press, 1980.

Ding Youyu, Zhou Hongli and Xu Zhu. Intensity determination of Steam Turbine. China Water Publish, 1985.

Tang Xinrun, Fang Qunbo,etc. ANSYS engineering apply tutorial (heat and electromagnetism section). China Railway Publishing House, 2003.

Xian Jiaotong University, Zhejiang University. Start-stop and test adjust of the large steam turbine. Publishing house of electronics industry, 1982.

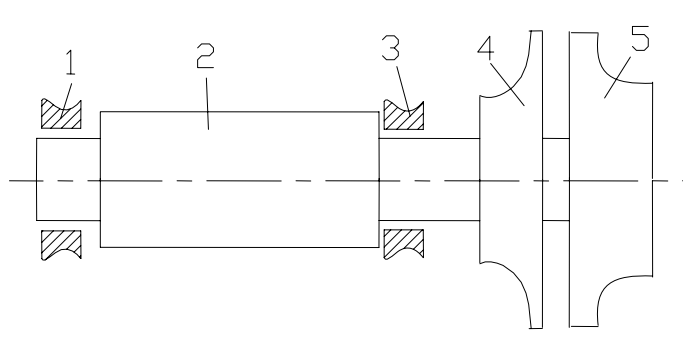

\section{3 bearing 2 .motor rotor}

4. compressor blade 5. turbo-expander wheel

Diagram 1.Structural diagram of gas turbine

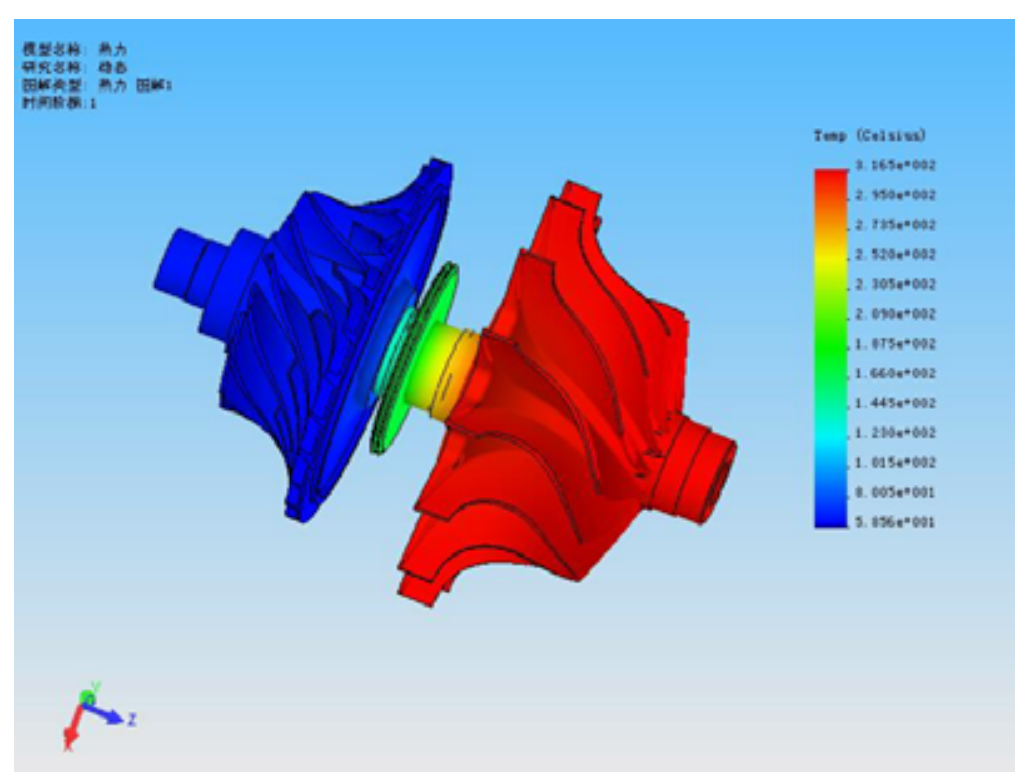

Diagram 2. the temperature field of rotor in steady 


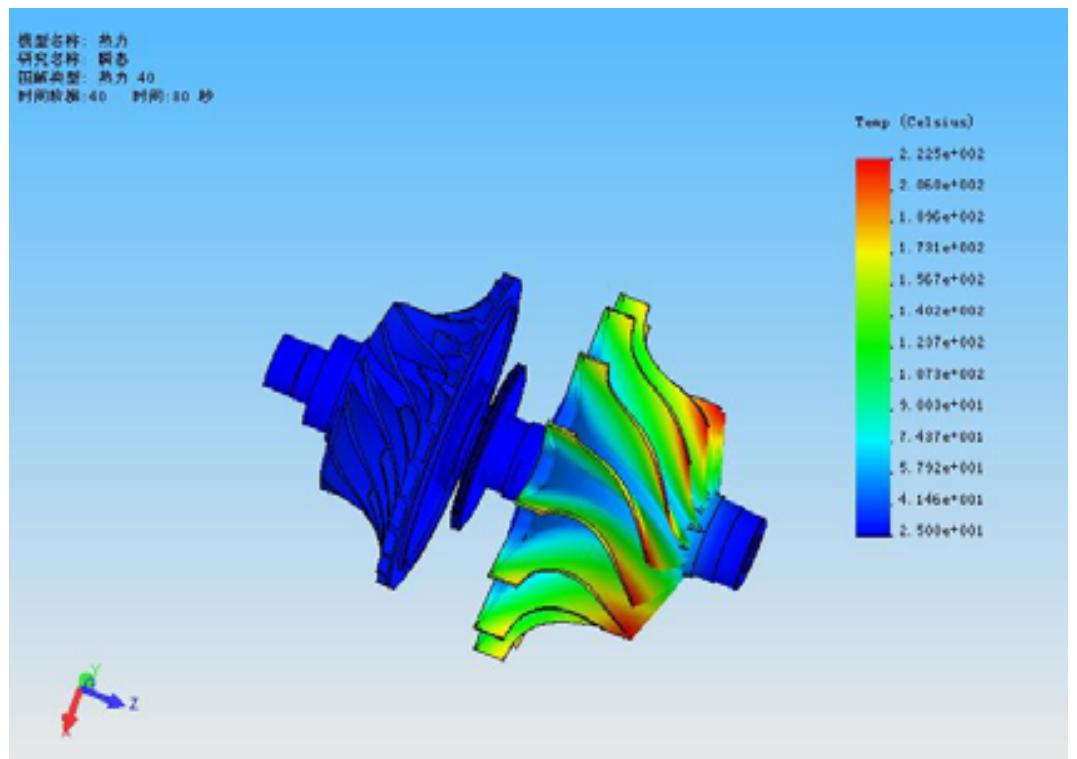

Diagram 3. the temperature field of rotor in transient (80s)

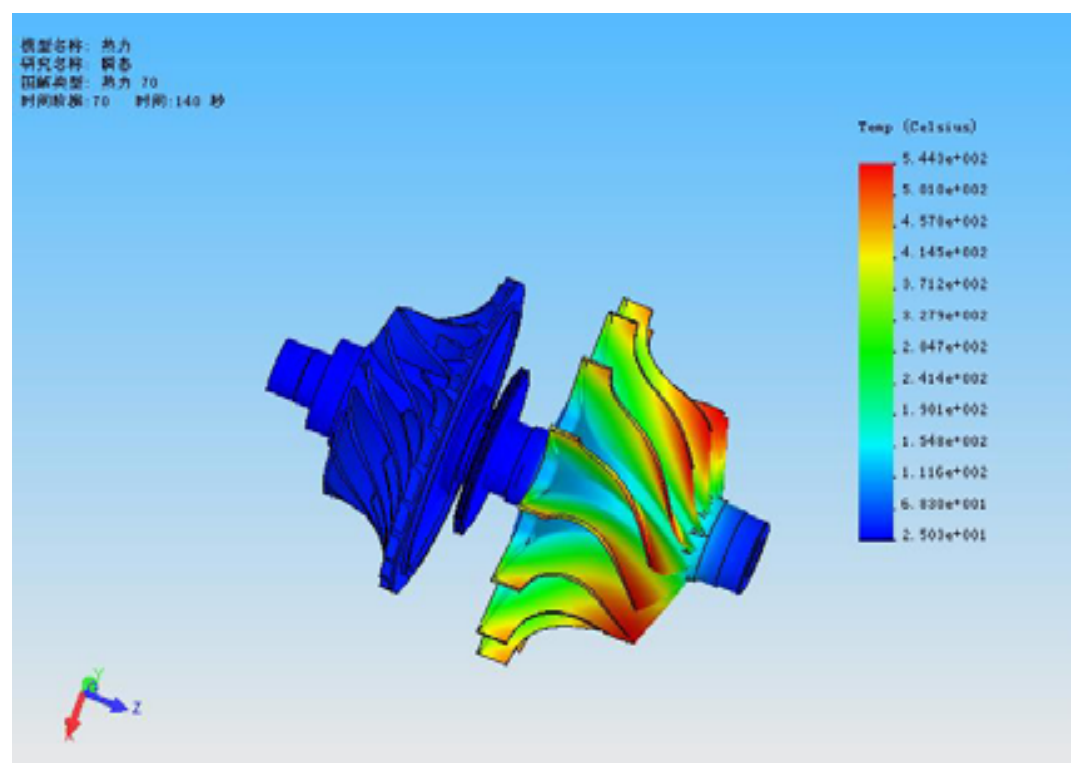

Diagram 4. the temperature field of rotor in transient (140s) 